\title{
OSCILLATION OF SOLUTIONS OF PARABOLIC EQUATIONS OF NEUTRAL TYPE
}

\author{
WEI NIAN LI AND BAO TONG CUI
}

\begin{abstract}
Some sufficient conditions for oscillation of solutions of parabolic differential equations of neutral type are obtained. These results are illustrated by some examples.
\end{abstract}

\section{Introduction}

Recently, the oscillation problem for the partial functional differential equation has been studied by many authors. We refer the reader to $[1,2,3]$ for parabolic equations and to $[4,5,6]$ for hyperbolic equations.

In this paper, we study the oscillation of parabolic differential equations of neutral type of the form.

$$
\begin{aligned}
& \frac{\partial}{\partial t}\left[b(t) u(x, t)-\sum_{s=1}^{r} \lambda_{s}(t) u\left(x, t-\tau_{s}\right)\right]=a(t) \Delta u(x, t)+\sum_{j=1}^{d} a_{j}(t) \Delta u\left(x, \rho_{j}(t)\right) \\
& -\sum_{h=1}^{l} q_{h}(x, t) u\left(x, \sigma_{h}(t)\right),(x, t) \in \Omega \times[0, \infty) \equiv G,
\end{aligned}
$$

where $\Omega$ is a bounded domain in $R^{n}$ with a piecewise smooth boundary $\partial \Omega$, and $\Delta u(x, t)=$ $\sum_{k=1}^{n} \frac{\partial^{2} u(x, t)}{\partial x_{k}^{2}}$.

Suppose that the following conditions hold:

(H1) $a, a_{j} \in C([0, \infty) ;[0, \infty)), j=1,2, \ldots, d$;

(H2) $q_{h} \in C(\bar{G} ;[0, \infty)), q_{h}(t)=\min _{x \in \bar{\Omega}} q_{h}(x, t), h=1,2, \ldots, l$;

(H3) $\rho_{j}, \sigma_{h} \in C([0, \infty) ;[0, \infty)), \lim _{t \rightarrow \infty} \rho_{j}(t)=\lim _{t \rightarrow \infty} \sigma_{h}(t)=\infty, \tau_{s}=$ const. $>0, j=$ $1,2, \ldots, d ; s=1,2, \ldots, r ; h=1,2, \ldots, l$;

(H4) $b \in C^{1}([0, \infty) ;(0, \infty)), \lambda_{s} \in C^{1}([0, \infty) ; R), b(t)$ is bounded, $b(t)-\sum_{s=1}^{r} \lambda_{s}(t) \geq 0$, $s=1,2, \ldots, r$.

We consider two kinds of boundary conditions:

$$
\frac{\partial u(x, t)}{\partial N}+g(x, t) u(x, t)=0,(x, t) \in \partial \Omega \times[0, \infty),
$$

Received February 24, 1999; revised May 5, 1999.

1991 Mathematics Subject Classification. 35B05, 35R10

Key words and phrases. Oscillation, parabolic equation, neutral type. 
where $N$ is the unit exterior normal vector to $\partial \Omega$ and $g(x, t)$ is a nonnegative continuous function on $\partial \Omega \times[0, \infty)$, and

$$
u(x, t)=0,(x, t) \in \partial \Omega \times[0, \infty) .
$$

Definition 1.1. A function $u \in C^{2}(G) \cap C^{1}(\bar{G})$ is called a solution of the problem (1), (2) (or (1), (3)) if it satisfies (1) in the domain $G$ along with the corresponding boundary condition.

Definition 1.2. The solution $u(x, t)$ of the problem (1), (2) (or (1), (3)) is said to be oscillatory in the domain $G=\Omega \times[0, \infty)$ if for any positive number $\mu$ there exists a point $\left(x_{0}, t_{0}\right) \in \Omega \times[\mu, \infty)$ such that $u\left(x_{0}, t_{0}\right)=0$ holds.

In the following two sections sufficient conditions are obtained for the oscillation of the solutions of the problem (1), (2) and (1), (3) in the domain $G$. We note that conditions for the oscillation of the solutions for $b(t)=1$ have been obtained in the works of Mishev and Bainov[1] and Cui[3].

\section{Oscillation of the problem $(1),(2)$}

\section{Theorem 2.1. If the neutral differential inequality}

$$
\left[b(t) V(t)-\sum_{s=1}^{r} \lambda_{s}(t) V\left(t-\tau_{s}\right)\right]^{\prime}+\sum_{h=1}^{l} q_{h}(t) V\left(\sigma_{h}(t)\right) \leq 0, t \geq 0
$$

has no eventually positive solutions. Then every solution $u(x, t)$ of the problem $(1),(2)$ is oscillatory in $G$.

Proof. Suppose to the contrary that there is a nonoscillatory solution $u(x, t)$ of the problem (1), (2) which has no zero in $\Omega \times\left[t_{0}, \infty\right)$ for some $t_{0} \geq 0$. Without loss of generality we may assume that $u(x, t)>0, u\left(x, t-\tau_{s}\right)>0, u\left(x, \rho_{j}(t)\right)>0$ and $u\left(x, \sigma_{h}(t)\right)>0$ in $\Omega \times\left[t_{1}, \infty\right), t_{1} \geq t_{0}, s=1,2, \ldots, r ; j=1,2, \ldots, d ; h=1,2, \ldots, l$.

Integrating (1) with respect to $x$ over the domain $\Omega$, we have

$$
\begin{aligned}
& \frac{d}{d t}\left[b(t) \int_{\Omega} u(x, t) d x-\sum_{s=1}^{r} \lambda_{s}(t) \int_{\Omega} u\left(x, t-\tau_{s}\right) d x\right]=a(t) \int_{\Omega} \Delta u(x, t) d x \\
& +\sum_{j=1}^{d} a_{j}(t) \int_{\Omega} \Delta u\left(x, \rho_{j}(t)\right) d x-\sum_{h=1}^{l} \int_{\Omega} q_{h}(x, t) u\left(x, \sigma_{h}(t)\right) d x, \quad t \geq t_{1} .
\end{aligned}
$$

Green's formula and (2) yield

$$
\int_{\Omega} \Delta u(x, t) d x=\int_{\partial \Omega} \frac{\partial u(x, t)}{\partial N} d S=-\int_{\partial \Omega} g(x, t) u(x, t) d S \leq 0,
$$


and

$$
\begin{aligned}
& \int_{\Omega} \Delta u\left(x, \rho_{j}(t)\right) d x=\int_{\partial \Omega} \frac{\partial u\left(x, \rho_{j}(t)\right)}{\partial N} d S \\
= & -\int_{\partial \Omega} g\left(x, \rho_{j}(t)\right) u\left(x, \rho_{j}(t)\right) d S \leq 0, t \geq t_{1}, \quad j=1,2, \cdots, d,
\end{aligned}
$$

where $d S$ is the surface element on $\partial \Omega$. Therefore,

$$
\begin{aligned}
& \frac{d}{d t}\left[b(t) \int_{\Omega} u(x, t) d x-\sum_{s=1}^{r} \lambda_{s}(t) \int_{\Omega} u\left(x, t-\tau_{s}\right) d x\right] \\
\leq & -\sum_{h=1}^{l} q_{h}(t) \int_{\Omega} u\left(x, \sigma_{h}(t)\right) d x, \quad t \geq t_{1} .
\end{aligned}
$$

Set $V(t)=\int_{\Omega} u(x, t) d x, t \geq t_{1}$, from (6) we have

$$
\left[b(t) V(t)-\sum_{s=1}^{r} \lambda_{s}(t) V\left(t-\tau_{s}\right)\right]^{\prime}+\sum_{h=1}^{l} q_{h}(t) V\left(\sigma_{h}(t)\right) \leq 0, \quad t \geq t_{1},
$$

which contradicts the assumption that (4) has no eventually positive solution. This completes the proof of Theorem 2.1 .

Theorem 2.2. Suppose that there exists a positive constant $\alpha$ and some $h_{0} \in$ $\{1,2, \ldots, l\}$ such that $q_{h_{0}}(t) \geq \alpha, \sigma_{h_{0}}^{\prime}(t) \geq 0$ and $\sigma_{h_{0}}(t) \leq t$. If $\lambda_{s}(t) \geq 0, s=1,2, \ldots, r$, and

$$
\limsup _{t \rightarrow \infty} \int_{\sigma_{h_{0}}(t)}^{t} \frac{q_{h_{0}}(s)}{b\left(\sigma_{h_{0}}(s)\right)} d s>1
$$

then every solution $u(x, t)$ of the problem (1), (2) is oscillatory in $G$.

Proof. Suppose to the contrary that there is a nonoscillatory solution $u(x, t)$ of the problem (1), (2) which has no zero in $\Omega \times\left[t_{0}, \infty\right)$ for some $t_{0} \geq 0$. Without loss of generality we may assume that $u(x, t)>0, u\left(x, t-\tau_{s}\right)>0, u\left(x, \rho_{j}(t)\right)>0$ and $u\left(x, \sigma_{h}(t)\right)>0$ in $\Omega \times\left[t_{1}, \infty\right), t_{1} \geq t_{0}, s=1,2, \ldots, r ; j=1,2, \ldots, d ; h=1,2, \ldots, l$.

As in the proof of Theorem 2.1 we obtain (7). Set

$$
\tau=\max _{1 \leq s \leq r}\left\{\tau_{s}\right\}, y(t)=b(t) V(t)-\sum_{s=1}^{r} \lambda_{s}(t) V\left(t-\tau_{s}\right), \quad t \geq t_{1}+\tau .
$$

From (12) and $\lambda_{s}(t) \geq 0, s=1,2, \ldots, r$, it follows that

$$
y^{\prime}(t)<0, y(t) \leq b(t) V(t) \text { for } t \geq t_{1}+\tau .
$$

Since $y(t)$ is ultimately strictly decreasing, then

$$
\lim _{t \rightarrow \infty} y(t)=L(\text { finite or }-\infty) .
$$


We will prove that $y(t)$ is bounded from below. Assume that $\lim _{t \rightarrow \infty} y(t)=-\infty$, then from the definition of $y(t)$ and (H4), we obtain that the function $V(t)$ is not bounded. Hence there exists a number $t_{2} \geq t_{1}+\tau$ satisfying

$$
V\left(t_{2}\right)=\max _{t_{1}+\tau \leq t \leq t_{2}} V(t), y\left(t_{2}\right)<0 .
$$

On the other hand, by (H4) we obtain

$$
y\left(t_{2}\right)=b\left(t_{2}\right) V\left(t_{2}\right)-\sum_{s=1}^{r} \lambda_{s}\left(t_{2}\right) V\left(t_{2}-\tau_{s}\right) \geq V\left(t_{2}\right)\left[b\left(t_{2}\right)-\sum_{s=1}^{r} \lambda_{s}\left(t_{2}\right)\right] \geq 0,
$$

which contradicts the inequality (11). Hence $L>-\infty$.

Integrating (7) over the interval $\left[t_{2}, t\right], t>t_{2}$, we have

$$
0<\alpha \int_{t_{2}}^{t} V\left(\sigma_{h_{0}}(s)\right) d s \leq \sum_{h=1}^{l} \int_{t_{2}}^{t} q_{h}(s) V\left(\sigma_{h}(s)\right) d s \leq-\int_{t_{2}}^{t} y^{\prime}(s) d s=y\left(t_{2}\right)-y(t) .
$$

Since $y\left(t_{2}\right)-y(t) \leq y\left(t_{2}\right)-L<\infty$, then $V(t) \in L_{1}\left(t_{0}, \infty\right)$. From condition (H4) we obtain that $y(t) \in \bar{L}_{1}\left(t_{0}, \infty\right)$, and hence $\lim _{t \rightarrow \infty} y(t)=0$, therefore,

$$
y(t)>0 \text { for } t \geq t_{3},
$$

where $t_{3} \geq t_{2}$ is a sufficiently large number.

Using (7) and (9), we obtain

$$
y^{\prime}(t)+\sum_{h=1}^{l} q_{h}(t) \frac{1}{b\left(\sigma_{h}(t)\right)} y\left(\sigma_{h}(t)\right) \leq 0, \quad t \geq t_{3} .
$$

It follows that

$$
y^{\prime}(t)+\frac{q_{h_{0}}(t)}{b\left(\sigma_{h_{0}}(t)\right)} y\left(\sigma_{h_{0}}(t)\right) \leq 0, \quad t \geq t_{3} .
$$

Integrating (14) from $\sigma_{h_{0}}(t)$ to $t$, we have

$$
y(t)-y\left(\sigma_{h_{0}}(t)\right)+\int_{\sigma_{h_{0}}(t)}^{t} \frac{q_{h_{0}}(s)}{b\left(\sigma_{h_{0}}(s)\right)} y\left(\sigma_{h_{0}}(s)\right) d s \leq 0, \quad t \geq t_{4}>t_{3} .
$$

Noting that $y^{\prime}(t)<0, \sigma_{h_{0}}(t) \leq t$ and $\sigma_{h_{0}}^{\prime}(t) \geq 0$, from (15) we get

$$
y(t)-y\left(\sigma_{h_{0}}(t)\right)+y\left(\sigma_{h_{0}}(t)\right) \int_{\sigma_{h_{0}}(t)}^{t} \frac{q_{h_{0}}(s)}{b\left(\sigma_{h_{0}}(s)\right)} d s \leq 0, \quad t \geq t_{4} .
$$

Therefore,

$$
\int_{\sigma_{h_{0}}(t)}^{t} \frac{q_{h_{0}}(s)}{b\left(\sigma_{h_{0}}(s)\right)} d s \leq 1-\frac{y(t)}{y\left(\sigma_{h_{0}}(t)\right)}<1
$$


And hence

$$
\limsup _{t \rightarrow \infty} \int_{\sigma_{h_{0}}(t)}^{t} \frac{q_{h_{0}}(s)}{b\left(\sigma_{h_{0}}(s)\right)} d s \leq 1,
$$

which violates the condition (8). This completes the proof of Theorem 2.2.

Theorem 2.3. Suppose that there exists a positive constant $\alpha$ and some $h_{0} \in$ $\{1,2, \ldots, l\}$ such that $q_{h_{0}}(t) \geq \alpha, \sigma_{h_{0}}^{\prime}(t) \geq 0$ and $\sigma_{h_{0}}(t) \leq t$. If $\lambda_{s}(t) \geq 0, s=1,2, \ldots, r$, and

$$
\liminf _{t \rightarrow \infty} \int_{\sigma_{h_{0}}(t)}^{t} \frac{q_{h_{0}}(s)}{b\left(\sigma_{h_{0}}(s)\right)} d s>\frac{1}{e},
$$

then every solution $u(x, t)$ of the problem (1), (2) is oscillatory in $G$.

Proof. As in the proof of Theorem 2.2 we have the inequality (14). On the other hand, by Lemma 1 of [7] we obtain that (14) has no eventually positive solution. This is a contradiction.

\section{Oscillation of the Problem (1), (3)}

The following fact will be used.

The smallest eigenvalue $\alpha_{0}$ of the Dirichlet problem

$$
\left\{\begin{array}{l}
\Delta \omega(x)+\alpha \omega(x)=0 \text { in } \Omega, \\
\omega(x)=0 \text { on } \partial \Omega
\end{array}\right.
$$

where $\alpha$ is a constant, is positive and the corresponding eigenfunction $\varphi(x)$ is positive in $\Omega$.

Theorem 3.1. If the differential inequality (4) has no eventually positive solutions, then every solution of the problem (1), (3) is oscillatory in G.

Proof. Suppose to the contrary that there is a nonoscillatory solution $u(x, t)$ of the problem (1), (3) which has no zero in $\Omega \times\left[t_{0}, \infty\right)$ for some $t_{0} \geq 0$. Without loss of generality we may assume that $u(x, t)>0, u\left(x, t-\tau_{s}\right)>0, u\left(x, \rho_{j}(t)\right)>0$ and $u\left(x, \sigma_{h}(t)\right)>0$ in $\Omega \times\left[t_{1}, \infty\right), t_{1} \geq t_{0}, s=1,2, \ldots, r ; j=1,2, \ldots, d ; h=1,2, \ldots, l$.

Multiplying both sides of $(1)$ by $\varphi(x)$ and integrating with respect to $x$ over the domain $\Omega$, we have

$$
\begin{aligned}
& \frac{d}{d t}\left[b(t) \int_{\Omega} u(x, t) \varphi(x) d x-\sum_{s=1}^{r} \lambda_{s}(t) \int_{\Omega} u\left(x, t-\tau_{s}\right) \varphi(x) d x\right] \\
= & a(t) \int_{\Omega} \Delta u(x, t) \varphi(x) d x+\sum_{j=1}^{d} a_{j}(t) \int_{\Omega} \Delta u\left(x, \rho_{j}(t)\right) \varphi(x) d x \\
& -\sum_{h=1}^{l} \int_{\Omega} q_{h}(x, t) u\left(x, \sigma_{h}(t)\right) \varphi(x) d x, \quad t \geq t_{1} .
\end{aligned}
$$


Green's formula and (3) yield

$$
\int_{\Omega} \Delta u(x, t) \varphi(x) d x=\int_{\Omega} u(x, t) \Delta \varphi(x) d x=-\alpha_{0} \int_{\Omega} u(x, t) \varphi(x) d x \leq 0,
$$

and

$$
\begin{aligned}
& \int_{\Omega} \Delta u\left(x, \rho_{j}(t)\right) \varphi(x) d x=\int_{\Omega} u\left(x, \rho_{j}(t)\right) \Delta \varphi(x) d x \\
= & -\alpha_{0} \int_{\Omega} u\left(x, \rho_{j}(t)\right) \varphi(x) d x \leq 0, \quad t \geq t_{1}, j=1,2, \cdots, d .
\end{aligned}
$$

Therefore,

$$
\begin{aligned}
& \frac{d}{d t}\left[b(t) \int_{\Omega} u(x, t) \varphi(x) d x-\sum_{s=1}^{r} \lambda_{s}(t) \int_{\Omega} u\left(x, t-\tau_{s}\right) \varphi(x) d x\right] \\
\leq & -\sum_{h=1}^{l} q_{h}(t) \int_{\Omega} u\left(x, \sigma_{h}(t)\right) \varphi(x) d x, \quad t \geq t_{1} .
\end{aligned}
$$

Set $V(t)=\int_{\Omega} u(x, t) \varphi(x) d x, t \geq t_{1}$, from (19) we obtain

$$
\left[b(t) V(t)-\sum_{s=1}^{r} \lambda_{s}(t) V\left(t-\tau_{s}\right)\right]^{\prime}+\sum_{h=1}^{l} q_{h}(t) V\left(\sigma_{h}(t)\right) \leq 0, t \geq t_{1},
$$

which contradicts the assumption that (4) has no eventually positive solution. This completes the proof of Theorem 3.1 .

It is not difficult to see that the following theorems are true.

Theorem 3.2. If all conditions of Theorem 2.2 hold, then every solution $u(x, t)$ of the problem (1), (3) is oscillatory in $G$.

Theorem 3.3. If all conditions of Theorem 2.3 hold, then every solution $u(x, t)$ of the problem (1), (3) is oscillatory in $G$.

\section{Examples}

Following are illustrative examples.

Example 4.1. Consider the parabolic equation

$$
\begin{aligned}
& \frac{\partial}{\partial t}\left[\left(1+e^{-t}\right) u(x, t)-u(x, t-2 \pi)\right]=2 e^{-t} \Delta u(x, t)+\left(2-e^{-t}\right) \Delta u\left(x, t-\frac{3 \pi}{2}\right) \\
& -e^{-t} u(x, t-\pi)-2 u\left(x, t-\frac{\pi}{2}\right),(x, t) \in(0, \pi) \times[0, \infty)
\end{aligned}
$$


with boundary condition

$$
\frac{\partial}{\partial x} u(0, t)=\frac{\partial}{\partial x} u(\pi, t)=0, t \geq 0
$$

Here $n=1, r=1, d=1, l=2, b(t)=1+e^{-t}, \lambda_{1}(t)=1, \tau_{1}=2 \pi, a(t)=2 e^{-t}$, $a_{1}(t)=2-e^{-t}, \rho_{1}(t)=t-\frac{3 \pi}{2}, q_{1}(x, t)=e^{-t}, q_{2}(x, t)=2, \sigma_{1}(t)=t-\pi, \sigma_{2}(t)=t-\frac{\pi}{2}$. It is easy to see that

$$
\limsup _{t \rightarrow \infty} \int_{\sigma_{2}(t)}^{t} \frac{q_{2}(s)}{b\left(\sigma_{2}(s)\right)} d s=\limsup _{t \rightarrow \infty} \int_{t-\pi / 2}^{t} \frac{2}{1+e^{\pi / 2-s}} d s=\pi>1 .
$$

Thus all the conditions of Theorem 2.2 are fulfilled. Then every solution of the problem $(20),(21)$ is oscillatory in $(0, \pi) \times[0, \infty)$. In fact, such a solution is $u(x, t)=\cos x \sin t$.

Example 4.2. Consider the parabolic equation

$$
\begin{aligned}
& \frac{\partial}{\partial t}\left[\left(1+e^{-t}\right) u(x, t)-u(x, t-2 \pi)\right]=\left(1+2 e^{-t}\right) \Delta u(x, t)+\left(2-e^{-t}\right) \Delta u\left(x, t-\frac{3 \pi}{2}\right) \\
& -\left(1+e^{-t}\right) u(x, t-\pi)-2 u\left(x, t-\frac{\pi}{2}\right),(x, t) \in(0, \pi) \times[0, \infty),
\end{aligned}
$$

with boundary condition

$$
u(0, t)=u(\pi, t)=0, t \geq 0 .
$$

It is easy to see that all conditions of Theorem 3.2 are fulfilled. Thus all solutions of the problem $(22),(23)$ are oscillatory in $(0, \pi) \times[0, \infty)$. In fact, such a solution is $u(x, t)=\sin x \sin t$.

\section{Acknowledgement}

The authors thank the referee for his comments of this paper.

\section{References}

[1] D. P. Mishev and D. D. Bainov, Oscillation of the solutions of parabolic differential equations of neutral type, Appl. Math. Comput., 28(1988), 97-111.

[2] X. L. Fu and W. Zhuang, Oscillation of neutral delay parabolic equations, J. Math. Anal. Appl., 191(1995), 473-489.

[3] B. T. Cui, Oscillation properties for parabolic equations of neutral type, Comment. Math. Univ. Carolinae, 33(1992), 581-588.

[4] B. T. Cui, Oscillation theorems of hyperbolic equations with deviating arguments, Acta Sci. Math. (Szeged), 58(1993), 159-168.

[5] B. T. Cui, Oscillation properties of the solutions of hyperbolic equations with deviating arguments, Demonstratio Math., 29(1996), 61-68.

[6] D. Bainov, B. T. Cui and E. Minchev, Forced oscillation of solutions of certain hyperbolic equations of neutral type, J. Comput. Appl. Math., 72(1996), 309-318. 
[7] B. T. Cui, Oscillation criteria for $n$-th order delay differential equations, Math. J. Toyama Univ., 13(1990), 1-8.

Department of Mathematics, Binzhou Normal College, Shandong 256604, China. 